\title{
Autonomía universitaria: \\ asunto público de interés privado
}

Revista Colombiana de Educación, N. ${ }^{\circ} 70$. Primer semestre de 2016 Bogotá, Colombia.

\section{//Autonomy at the University \\ Private-Concern, Public Issue}

\section{//Autonomia universitária: assunto público de interesse privado}

\section{Alcira Aguilera Morales*}

\begin{abstract}
Doctora en Estudios Latinoamericanos de la UNAM, magíster en Desarrollo Educativo y Social del Cinde/UPN. Docente investigadora del programa de licenciatura en Educación Infantil de la Universidad Pedagógica Nacional. Bogotá, Colombia. Integrante del grupo de Investigación "Sujetos y nuevas narrativas en la investigación y enseñanza de las ciencias sociales". Correo electrónico: aamorales@pedagogica.edu.co
\end{abstract}

\section{Resumen}

El presente artículo es producto de la investigación doctoral "Acciones colectivas en defensa de la educación pública: Colombia-México (1987-2007)". En él se presenta específicamente la configuración de la noción de autonomía universitaria desde el sentido de lo público a partir de dos experiencias universitarias: el caso de la UNAM en México y el de la Universidad Nacional de Colombia. Ello responde a la preocupación por lo polisémico del concepto, las tensiones y confusiones que se presentan cuando se alude al mismo y la necesidad de rediseñarlo de cara a la realidad universitaria actual. Para ello se abordan tres concepciones que a modo de antinomias explican la configuración del sentido de la autonomía universitaria en relación con la Iglesia, el Estado y el Mercado.

\section{Abstract}

This article is a result of the doctoral research "Collective Actions in Defense of Public Education: Colombia-Mexico (1987-2007)". It specifically shows the configuration of the concept of university autonomy from a public perspective, based upon two college experiences, the case of the UNAM in Mexico and the National University of Colombia. This responds to the concern about the polysemy of the concept, as well as the tensions and confusions that arise when referring to it and the need to redesign it in regards to the current university reality. For this reason, the study addresses three conceptions that, as contradictions, explain the configuration of the sense of university autonomy in relation to the Church, the State and the market.

\section{Resumo}

O seguinte artigo é produto da pesquisa doutoral "Acciones colectivas en defensa de la educación pública: Colombia-México (1987-2007)". Nele apresenta-se especificamente a configuração da noção de autonomia universitária, desde o sentido do público a partir de duas experiências universitárias, o caso da UNAM em México e da Universidad Nacional de Colombia. Isso responde à preocupação pelo polissêmico do conceito, as tenções e confusões que apresentam-se quando se refere ao mesmo e a necessidade de redesenha-lo de face à realidade universitária atual. Para isto se têm três concepções que a modo de antinomias explicam a configuração do sentido da autonomia universitária em relação com a Igreja, o Estado e o Mercado.

\section{Palabras clave}

Autonomía universitaria, educación pública, universidad pública, UNAM, Universidad Nacional de Colombia

\section{Keywords}

Autonimy at the University, public education, public university, UNAM, Universidad Nacional de Colombia

\section{Palavras chave}

Autonomia universitária, educação pública, universidade pública, UNAM, Universidad Nacional de Colombia 
La autonomía universitaria es, a la vez, memoria y futuro, condición y límite, fortaleza y oportunidad. Adrián Acosta Silva

El debate sobre el sentido público de la autonomía universitaria está ligado a la manera como en los diferentes estados latinoamericanos se configuraron la educación y la universidad pública. Este proceso estuvo ligado a diversos elementos: la relación que mantuvo el Estado con la Iglesia en su proceso de constitución, pues en varias investigaciones (Aguilera, 2013; Levy, 1995) se ha constatado que en el caso mexicano al primar la consolidación de un Estado laico se favoreció el despliegue de un sentido fuerte de lo público en el ámbito educativo y en otros sectores sociales. Por su parte, en el caso colombiano prevaleció la consolidación de un Estado confesional que priorizó el desarrollo de la moral y el orden católico sobre el desarrollo de lo público en todas las esferas sociales ${ }^{1}$. De igual manera la influencia de los idearios sobre la "instrucción pública" en torno a principios como la laicidad, igualdad, universalidad y gratuidad, retomados de la Revolución francesa y su lema "Libertad, igualdad y fraternidad" (Condorcet, 2007), tuvieron diferentes ecos en cada caso.

A grandes rasgos, se puede destacar que la tradición liberal, a partir de la disputa librada a lo largo del siglo xix en México, finalmente logró instaurar con Benito Juárez un Estado laico, civil, reformista que, a su vez, concibió los asuntos de la educación superior desde esta óptica. De este modo se hizo de la educación pública y de la unam el escenario de la educación laica, nacional, científica y gratuita. Este ideario se retomó posteriormente en la Constitución Política Mexicana de 1917, de allí que los hitos fundacionales de la educación pública en México se han mantenido, no solo por registrarse en códigos legales sino en el código cultural; es decir, en prácticas, creencias, valores y discursos que han hecho que el sinónimo de lo público en la educación sea la gratuidad, la laicidad y la autonomía. Por el contrario, en el caso colombiano se encuentra una herencia impostada, en la

1 En el libro de mi autoría Subjetividades politicas en movimiento(s). La defensa de la universidad pública en Colombia y México se desarrolla con amplitud cómo se dio cada uno de estos procesos en ambos países, analizando la configuración del ideario de lo público en la educación y sus repercusiones en las concepciones de gratuidad, universalidad y laicidad. 
que existe un gran vacío desde los hitos fundacionales, al no contar con momentos rememorados de nuestra historia que de manera clara hagan mención a la existencia de ese ideario público. De igual forma, no se acude a un marco legal, una ley o un código legislativo que sirvan de marco jurídico para defender una idea de educación pública, pues la Constitución Política de 1991, así como la Ley 30 de 1992, la asumen como servicio y no definen con claridad su universalidad, obligatoriedad, gratuidad y autonomía (Aguilera, 2011).

Con este breve panorama sobre la configuración de las universidades públicas estudiadas podemos adentrarnos en el aspecto que nos convoca: la mirada comparada sobre la configuración de la autonomía universitaria como asunto público que está en permanente disputa con los intereses privados.

\section{La autonomía universitaria y lo público}

Los actuales debates sobre la esfera de lo público descentran la relación entre lo público y el Estado. Desde esta perspectiva, hablar de educación, salud, universidad, entre otros aspectos que atañen a lo público, no alude directamente a la responsabilidad del Estado en su consecución y garantía. En su lugar se hace la distinción entre lo público estatal y lo público no estatal, o también denominado tercer sector. Así, esta perspectiva supone dos condiciones: “1) La preeminencia del ámbito de lo público como campo y objeto de acción, y 2) la naturaleza voluntaria, libre, del asociacionismo que lo genera" (Varela, 2005, p. 43). En ambas condiciones lo público no estatal está referido a la fuerza de la solidaridad fundante y no a lo público estatal fundado en la coacción universalista de las leyes. Sin embargo, esta postura no reconoce el debate histórico sobre el que se cimentó la educación pública y con ella la autonomía universitaria, pues en ambos aspectos fue fundamental la relación establecida con el Estado.

Por esta razón, retomamos la distinción clásica de lo público y lo privado, ya que desde allí vuelve a cobrar sentido la discusión sobre la autonomía universitaria. Veamos: lo público está asociado a la "pertenencia de todos, con base en el principio de jurisdiccionalidad de la inclusión" (Varela, 2005, p. 40). En oposición a lo público se define lo privado, en palabras de Arendt;

[...] en la actualidad llamamos privada a una esfera de intimidad cuyo comienzo puede rastrearse en los últimos romanos, apenas en algún período de la antigüedad griega, y cuya peculiar multiplicidad y variedad era desconocida en cualquier período anterior a la Edad Media. En el sen- 
timiento antiguo, el rasgo privativo de lo privado, indicado en el propio mundo, era muy importante; literalmente significaba el estado de hallarse desprovisto de algo, incluso de las más elevadas y humanas capacidades. (1993, p. 49)

De esta manera, lo privado se entiende como privación o exclusión, sería entonces desde una posición de negación. Sin embargo, Varela (2005) también encuentra en la definición negativa de lo privado un sentido positivo que alude al disfrute continuado y asegurado de la posesión de aquello que se les niega a los demás.

De acuerdo con ello, en esta investigación asumimos la distinción que hace Rabotnikof (1997), para quien lo público se refiere a lo colectivo, al bien o interés común, en oposición al interés individual o particular. Esta distinción, en términos políticos, opone aquello que es de interés público a lo que es de interés privado. Aquí lo público sigue vinculado con la idea de Estado, por arraigar el bien común y general; por ello, desde esta postura se rastrea la configuración de la autonomía universitaria en la unAm y la Universidad Nacional de Colombia, para mostrar cómo la autonomía emerge como oposición al interés privado representado en un credo (la Iglesia), el monopolio del poder (el Estado), en el usufructo y la mercantilización de la vida universitaria (el Mercado).

Desde esta mirada se reconoce que aludimos a un concepto histórico, que está constituido por contenidos que se "van confirmando y a la vez redefiniendo en cumplimiento de sus funciones relativas a la creación de conocimiento y su cambio en el tiempo y en la sociedad nacional y universal" (Ordorika, 2010, p. 86). De esta manera, aunque la autonomía de la que podemos hablar hoy no es la autonomía que se debatía hace un siglo, podemos mencionar los aspectos que la animan, que siguen latentes en el escenario universitario actual, que se pone en disputa entre los diferentes actores sociales y en los diferentes contextos de época.

\section{La autonomía como antinomia del poder hegemónico}

Un ejercicio interesante que puede ayudar a entender cómo se constituye la autonomía universitaria consiste en rastrear históricamente las luchas y oposiciones que las universidades visibilizan en torno a los poderes hegemónicos, pues allí encontramos claves para desentrañar por qué el interés privado, es decir de pocos, se opone a aquella autonomía que representa el interés general, 
o del bien común. Ello quiere decir que la autonomía universitaria se empieza a dibujar y a consolidar por su capacidad de opción frente al poder instaurado.

Este ejercicio contribuye a identificar cómo se dan estas oposiciones en cada caso estudiado y a partir de allí establecer las concepciones de autonomía universitaria que prevalecen. De esta manera planteamos la constitución de la autonomía como antinomia de tres poderes hegemónicos: Iglesia, Estado y Mercado.

\section{Autonomía: entre un credo y el libre pensamiento}

Es innegable el papel que cumplieron la Iglesia y diferentes órdenes religiosas en la creación de los primeros centros educativos y universidades de América Latina y Europa. No obstante, las primeras universidades ${ }^{2}$ representadas en corporaciones estudiantiles gozaban de un poder considerable aunque nunca fueron completamente autónomas (González, 2001). Así, por ejemplo "La universidad de Bolonia surgió del impulso de la comunidad estudiantil a finales del siglo xII; sacó provecho de la rivalidad entre el Papa y el emperador, y consiguió importantes privilegios y una jurisdicción amplia" (Luna Díaz [1987], citada en Ordorika, 2006, p. 21). En este mismo rastreo se menciona que

2 Con el término universitates se refería, originalmente, a las comunidades, los gremios o las corporaciones estudiantiles que tenían un reconocimiento público en el siglo XII (véase Ordorika, 2006). las universidades de Bolonia y París presentaron fuertes disputas con la Iglesia y la Corona en función de lograr un mayor nivel de libertad, en vista del esfuerzo de la Iglesia y de las autoridades locales por ejercer el control sobre ellas. Así, la libertad de acción y elección de las universidades empieza a confrontar lo que la Iglesia o la Corona querían hacer de ellas. Este es un primer registro de la autonomía universitaria que marcaría el inicio de la búsqueda de independencia por parte de la universidad con respecto a los intereses representados en la Corona.

Estas disputas se sentirían en territorio latinoamericano, guardando las respectivas proporciones y sentidos de la universidad latinoamericana, seis siglos más tarde. Desde esta perspectiva los antecedentes de la demanda por la autonomía universitaria, los encontramos en el Nuevo Reino de Granada - pese a que este periodo antecede a la concepción de universidad pública moderna-, registrados en la lucha estudiantil por adquirir autonomía e independencia de la Corona, al exigir fundamentalmente el acceso a los conocimientos científicos.

Vemos que un rasgo de la autonomía universitaria está asociado a la producción de un conocimiento libre de los dogmas impuestos por el poder hegemónico y útil a la sociedad del momento. Esto se refleja en el movimiento social --estudiado por Diana Soto-- Ilevado a cabo en los colegios mayores de San Bartolomé y el Rosario, gestado fuera de las aulas, 
principalmente en las tertulias de Santa Fe de Bogotá en el siglo XVIII $(1791,1794$ y 1796). De acuerdo con Soto (1999), la tertulia más influyente al respecto fue la de Antonio Nariño, en la cual se trataban asuntos políticos, sociales y científicos, seguida de la del grupo de José Celestino Mutis. En este ambiente político los estudiantes solicitaban que se les diera la ciencia útil, que ya conocían por la época de la aplicación del plan Moreno y Escandón ${ }^{3}$ presentado en 1768, conocido como el proyecto de universidad pública para el gobierno virreinal, propuesta que desde luego fue atacada por el clero. Para 1774 Moreno y Escandón, siendo

[...] Director Real de Estudios, nuevamente presentó un Plan de estudios que orienta la enseñanza secundaria y superior hacia una concepción más práctica y hacia las ciencias experimentales. Criticó la educación que impartían los religiosos, pues decía que no manejaban conceptos científicos profundos y que sólo hacían uso de tratadistas herederos de la tradición escolástica medioeval (trívium y cuadrivium), desconocían las teorías de Copérnico y alejaba a la educación de los movimientos intelectuales de la época inspirados en la llustración. Propugnó una Universidad Pública orientada con espíritu secular; en la que los seglares se entendieran de los asuntos educativos. (Hernández de Alba, 2009, p. 197)

El Gobierno virreinal, al detectar que durante la última década del siglo xvIII la élite criolla prefiguraba un proyecto nacionalista que se apoyaba en la filosofía ilustrada, inició los controles en la enseñanza y reprimió a los catedráticos que impartían la nueva ciencia (Aguilera, 2010; Soto, 1999). En esta disputa vemos un primer intento por separar la labor de colegios mayores o instituciones de formación superior y la intervención de la Corona al no permitir el desarrollo del conocimiento científico y del libre pensamiento en los claustros educativos.

De igual manera en el siglo xvı, en la Nueva España (actual México), se gesta la Real Universidad en 1551, bajo la dirección del virrey y la Real Audiencia, dirección que no estuvo exenta de disputas y tensiones:

3 Francisco Antonio Moreno y Escandón presentó el documento Método provisional de estudios de Santa Fé de Bogotá para los colegios (1774). Este documento antecede los debates de Condorcet sobre la educación pública presentados en 1791. Fue uno de los notificadores y ejecutor de la real cédula de Carlos III que ordenaba la expulsión de los jesuitas de todos los dominios hispanos. (Véase Hernández de Alba, 2009). 
El rey como patrono universal de la iglesia americana, podía haber puesto su universidad novohispana al cuidado de su arzobispo, como lo hizo cuando fundó la Universidad Real de Granada. Pero en 1551 no existía aún una iglesia consolidada en la Nueva España, pues las órdenes religiosas estaban volcadas a la evangelización de los naturales, mientras que los obispos tenían escaso poder y riquezas. En tales circunstancias se explica que el monarca encomendara el cuidado de la universidad al virrey y la Audiencia; la decisión tenía algo de coyuntural y esa circunstancia un tanto atípica para la época, sería fuente de incontables conflictos. (González, 2001, p. 25)

Esta naciente universidad retomó la organización de los saberes tradicionales de las universidades medievales: Teología, Cánones, Leyes, Medicina y Artes. Estos conocimientos servían de base para la reproducción de las élites criollas, así como para sostener el poder de la Corona. Así, las disputas encarnadas en cómo y quién gobierna los centros universitarios será una de las disputas que en el futuro consagrará la autonomía universitaria.
Sin embargo, en el caso mexicano se encuentra que una de las primeras manifestaciones en torno a la autonomía universitaria se dio a finales del siglo XIX, específicamente en el año de 1875, cuando el movimiento estudiantil denominado Universidad Libre luchó en oposición a la herencia colonial que mantenía presa a la Escuela Nacional de Medicina ${ }^{4}$, haciendo énfasis en la apertura a un sistema educativo liberal y libre del Estado. Al respecto, Alvarado (1999) menciona que este movimiento se adelantó en México entre el 21 de abril y el 8 de mayo de 1875 ,

$$
\begin{aligned}
& \text { Cuando Francisco Ortega, } \\
& \text { director de la Escuela } \\
& \text { Nacional de Medicina } \\
& \text { expulsó a dos estudiantes } \\
& \text { de ese plantel por haber } \\
& \text { provocado que el resto de } \\
& \text { los jóvenes se negaran a } \\
& \text { asistir a clases en señal } \\
& \text { de inconformidad contra } \\
& \text { los métodos pedagógicos } \\
& \text { del Dr. Rafael Lavista. [...] } \\
& \text { La huelga escolar se bau- } \\
& \text { tizó con la denominación } \\
& \text { de "Universidad Libre", } \\
& \text { para así diferenciarla de }
\end{aligned}
$$

4 Para este periodo no existía universidad como tal, puesto que el liberalismo radical representado con Benito Juárez erradicó la universidad, desde entonces se crearon escuelas nacionales profesionales, que reunían las profesiones liberales, entre ellas: Instrucción Secundaria de Personas del Sexo Femenino; Estudios Preparatorios; Jurisprudencia; Medicina, Cirugía y Farmacia; Agricultura y Veterinaria; Ingenieros; Naturalistas; Bellas Artes; Música y Declamación, y Comercio. Además, existían una escuela normal, una de artes y oficios y una más para sordomudos. (Véase Alvarado, 2001) 
su antecesora colonial, esta sería libre, independiente del Estado. En este se destacó la insistente demanda tanto de maestros como de alumnos, de una apertura liberal del sistema educativo, el que a juicio de estos últimos continuaba regido por 'antiguas prácticas, funesto del legado de la dominación española’. (p. 70)

Se considera que uno de los logros más importantes del movimiento es la creación de una enseñanza independiente del Estado. Este será otro de los rasgos de la autonomía universitaria que siempre ha tensionado la relación Universidad-Estado y es la permanente lucha porque los campus universitarios definan qué ciencia, qué conocimientos y qué posibilidades de pensamiento ofrecen, atendiendo las realidades y necesidades sociales de cada contexto, aspecto que suele ser intervenido por el Estado, que como se sabe termina respondiendo a los intereses de grupos minoritarios.

En síntesis, podemos mencionar que la producción de conocimiento, libre de dogmas, pero además de un conocimiento capaz de superar la herencia colonial, ha sido una de las disputas que define la autonomía universitaria. Ello en gracia a que un encargo histórico de las universidades públicas es contribuir al desarrollo de las naciones, proyecto que en muchos casos dista de los planes de la Iglesia o de los Estados, instituciones que por demás concentran el interés de las élites, no el beneficio de las mayorías. En este horizonte de sentido se inscribe por tanto la libertad de enseñanza y de cátedra, asunto que no es reductible a la importante labor de producir conocimiento/pensamiento de acuerdo con los proyectos y destinos de los pueblos latinoamericanos, libre de credos, libre por fin de la herencia colonial.

\section{La autonomía como disputa del gobierno propio y del gobierno de otros}

Un principio que contribuyó a la configuración de la educación y la universidad publica, producto del surgimiento de los Estados nacionales en América Latina, fue su carácter laico, ya que la ruptura Iglesia-Estado manifestaba que la nueva educación estaría al servicio del segundo; se buscaba romper de raíz lo fue la educación confesional, religiosa, al servicio de las "coronas" y la fe católica. Solo la educación laica garantizaría la formación 
del nuevo hombre, como ciudadano partícipe de un nuevo orden civil ${ }^{5}$. Además, la educación laica también se asociaba a la formación en las ciencias y la ampliación de los campos del conocimiento como manera de superar la tradición religiosa. De acuerdo con García Laguardia, aludiendo al caso mexicano,

Las universidades coloniales desaparecieron vio-lenta o lentamente a lo largo del siglo xix y fueron remplazadas por escuelas federales, ligadas a los programas de unidad nacional de los gobiernos centrales. Se abandonó la teología como centro de interés y se la sustituyó por las profesiones liberales. Mientras los regímenes liberales mantuvieron su pureza el Estado ejerció un amplio monopolio educativo y cerró las puertas a las diferentes órdenes religiosas. (Citado en Levy, 1995, p. 89)

Es decir que el nuevo sistema de educación pública nace de su fuerte alianza con el Estado, ya que ayudaría a legitimar una nueva historia del poder, de la mano de una educación laica y liberal. Esta educación implicaría que la alianza fuese a su

5 Sin embargo, en el caso colombiano se encuentra una fuerte herencia colonial y por tanto el mantenimiento de muchas de las cátedras coloniales en las primera universidad post-independencia. (Véase Aguilera M., 2011). vez motivo de tensión pues ahora el control lo ejercerían los Estados sobre las que serían las primeras universidades públicas.

El siglo xIX sería escenario de esta disputa entre Universidad y Estado, principalmente reclamando la no interferencia del Estado en los asuntos de la vida universitaria, en sus formas de gobierno y en la toma de decisiones que regulan la vida académica y la producción de conocimiento científico y crítico.

En el caso de la UnAm, en Justo Sierra ${ }^{6}$, intelectual de la época, se encuentra gran parte de la concepción de autonomía, ya que siendo secretario de Instrucción Pública trabajó por la separación de la educación y el Estado, e insistió en que este seguiría asumiendo su responsabilidad económica para con lo educativo, a la vez que defendía la necesidad de establecer la libertad de enseñanza. El proyecto de la Universidad Nacional de México presentado por él tuvo muchos detractores debido a que durante todo el siglo xIx el liberalismo radical sepultó la institución universitaria por considerarla dogmática, herencia colonial

6 Justo Sierra (1848-1912), poeta, periodista, historiador y político mexicano, fundador de la Universidad Nacional Autónoma de México y defensor de la educación primaria obligatoria y gratuita en México. Fue varias veces diputado en el congreso de la Unión, magistrado de la Suprema Corte de Justicia y ministro de Instrucción Pública en varios periodos del porfiriato. 
y enemiga de la ciencia. Pese a estos prejuicios, Sierra defendió una nueva universidad, sin árbol genealógico, sin la intromisión del Estado. En sus palabras:

El Estado tiene una alta misión política, administrativa y social; pero en esa misión misma hay límites, y si algo no puede ni debe estar a su alcance, es la enseñanza superior, la enseñanza más alta. La enseñanza superior no puede tener, como no tiene la ciencia, otra ley que el método; esto será normalmente fuera del alcance del gobierno. Ella misma, es decir, los docentes que forman por sus conocimientos esta agrupación se llamará Universidad Nacional [y] será la encargada de dictar las leyes propias, las reglas propias de su dirección científica; y no quiere decir esto que el gobierno pueda desentenderse de ellas, ni impedir que lleguen a su conocimientos, ni prescindir, en bien del Estado, el derecho de darles su aprobación última. (Sierra, 1910, pp. 420-421)

En este fragmento, que forma parte del discurso de creación de la Universidad Nacional de México, es evidente la idea de la autonomía universitaria por cuanto se reconoce que es la universidad, atendiendo a su propia comunidad docente, la encargada de establecer sus propias leyes así como las orientaciones para su desarrollo científico. El lugar que le concede al Estado es el de reconocer sus límites en el desarrollo de la vida universitaria, continuando con su misión financiadora y administrativa. De esta manera es evidente que el proyecto de universidad pública estaba orientado para ser gobernado por los propios docentes, y aunque no mencionaba a la comunidad estudiantil, se pensaba en una universidad con gobierno propio y administrativamente financiada por el Estado.

Este rasgo de la autonomía universitaria antecede el Movimiento de Reforma Universitaria de Córdoba, Argentina, que se fundó en un fuerte sentimiento anticlerical (Tunnerman, 1976), debido a que mientras la universidad de la Plata se fundaba bajo la "impronta del positivismo", la de Córdoba tenía fuertes imposiciones dogmáticas (Cuneo, 1988). De ahí que el movimiento luchara contra un régimen administrativo, contra un concepto de autoridad y de método docente, tal como se expresó en el Manifiesto de Córdoba de 1918: 
La Federación Universitaria de Córdoba se alza para luchar contra este régimen y entiende que en ello le va la vida. Reclama un gobierno estrictamente democrático y sostiene que el demos universitario, la soberanía, el derecho de darse el gobierno propio radica principalmente en los estudiantes. (Manifiesto de Córdoba, 1918)

Efectivamente la reforma tendría su eco en toda América Latina y pondría un acento importante en la constitución de un "gobierno propio", asunto que siempre tensiona las relaciones entre universidad y Estado, ya que al contar con la financiación estatal se da por hecho que el Estado tiene derecho a intervenir en el gobierno y las decisiones propias de la vida universitaria. Se confunden de esta manera las labores de vigilancia y control sobre el destino de los recursos públicos de las universidades con la autonomía universitaria; al decir de Acosta (2008), no se separan las exigencias administrativas de las responsabilidades académicas.

Retomando el caso de la UNAM se encuentra un momento memorable en 1929 cuando, después de una huelga estudiantil que duró más de dos meses ${ }^{7}$, el presidente Emilio

7 Este movimiento se desató tras la aplicación de un nuevo sistema de reconocimiento de la Facultad de Derecho y de un nuevo plan de estudios de tres años en la Escuela Nacional Preparatoria (anteriormente eran dos años). El
Portes Gil, con la aprobación del Congreso, otorgó la autonomía universitaria a la Universidad Nacional de México (Marsiske, 2001; Ordorika, 2006). Esta autonomía no era objeto de reclamos ni disputas, pero sirvió de comodín para apaciguar los ánimos estudiantiles. Incluso algunos analistas definen esta decisión como un castigo más que como un reconocimiento (Camboni, 1998; García Cantú, 1988). Siguiendo a Marsiske (2001), esta era considerada una autonomía relativa por cuanto el Ejecutivo mantenía controles sobre la vida universitaria, por ejemplo el presidente se reservó el derecho a presentar una terna ante el Consejo Universitario para el nombramiento del rector e incluso para influir en la elección de rectores que no pertenecieran a la máxima casa de estudios. En este contexto el gobierno universitario estaba compuesto de la siguiente manera:

La máxima autoridad de la Universidad era el Consejo Universitario, integrado por el rector, el secretario de la Universidad, y los directores de

pliego de peticiones estudiantil incluiría "la destitución de del secretario de educación pública y del subsecretario de educación; la renuncia del jefe de la policía; la minuciosa investigación para encontrar al culpable de la represión del 23 de mayo; mayor participación de los estudiantes en el Consejo Universitario; Creación de un Consejo Técnico para las escuelas técnicas, equivalente al Consejo universitario; creación de un Consejo de Escuelas Normales; Reincorporación de las secundarias a la Preparatoria y Elección del rector de la Universidad por el presidente de la República de una terna que le presenta el Consejo Universitario" (Marsiske, 2001, p. 156). 
las facultades, escuelas e instituciones universitarias como miembros ex oficio; los miembros electos del consejo serían dos profesores titulares por cada una de las facultades y escuelas, dos alumnos y una alumna delegados de la Federación Estudiantil, y un delegado de la Secretaría de Educación Pública con voz informativa únicamente. (Marsiske, 2001, pp. 159-160)

Con estos antecedentes aún no podemos hablar de un gobierno universitario propio. Como vemos, se encuentra la interferencia del Gobierno representada en la participación de la Secretaría de Educación Pública, así sea solo con su voz. En 1933, además de lograr una lucha por la autonomía, la universidad adquiere su impronta definitiva como Universidad Nacional Autónoma de México, en razón a que en dicho año se impulsa el proyecto gubernamental de establecer una educación socialista, desde una filosofía orientada por el materialismo histórico, en medio de la campaña presidencial de Lázaro Cárdenas (Ramírez, 2001). La propuesta tenía tanto seguidores como detractores, pero la postura que se impuso fue la que defendía una educación libre de ideologías, ya que un importante sector estudiantil leía en esta propuesta una imposición sectaria que atentaba contra la autonomía y la libertad de cátedra. Así las cosas, la universidad adquirió una autonomía total, dejó de ser nacional y perdió el apoyo financiero estatal, en castigo por oponerse al establecimiento de una educación socialista para toda la nación.

Finalmente en el año de 1945, con una Ley Orgánica que sustituía la anterior y que separaba la labor científica de la política, se consolidó la noción de autonomía universitaria en la unam. En opinión de García Cantú (1988), esta ley representa la mayor expresión de la autonomía, al separar la actividad universitaria de los intereses políticos y del Estado, ya que estos buscan hacer de la universidad un campo de adiestramiento. De modo que la autonomía se asume desde la idea de que la universidad es el campus de producción de conocimiento técnico científico, no de reproducción de la vida política. De acuerdo con Ordorika (2006) esta noción se propuso hacer de la universidad una institución apolítica.

En el caso de la Universidad Nacional de Colombia encontramos un proceso bien distinto, marcado por la fuerte injerencia de la Iglesia y con ella de lo estipulado en la Constitución Política de Colombia de 1886 — que rigió hasta 1991—y del Concordato 
de 1887, en el que se estipuló que la Iglesia era quien administraba y tenía la potestad para definir qué enseñar en todos los niveles educativos, incluyendo el universitario (Aguilera, 2010). Con este panorama las luchas estudiantiles de la década de los veinte reclamaban la autonomía universitaria en torno a las mejoras académicas o al cambio de autoridades (Archila, 1999). En esta década se encuentra una idea de autonomía universitaria muy enraizada en el movimiento reformista cordobés:

[La Universidad que nosotros queremos] $\mathrm{Ha}$ de ser una universidad independiente, libre, dueña de sus propios recursos, libre de toda tutela, que pueda orientarse en cualquier instante de acuerdo con las sugestiones de la ciencia y de la vida. Reflejo del alma nacional, contradictoria e inquieta, pero jamás del sentir exclusivo de un poder político. Que se desenvuelva en su organización con autonomía verdadera, que se dicte sus leyes por un acuerdo entre el profesorado y los estudiantes, que jamás pueda turbar su marcha el temor de una imposición extraña. (Arciniegas, 1923, citado en Cuneo, 1988, p. 60)
En ella se reafirma la necesidad de un gobierno universitario propio sin la interferencia del Estado o gobierno de turno. Un gobierno propio que esté orientado por estudiantes y profesores, en las palabras de Germán Arciniegas $^{8}$, se trata de una

\section{[...] relación entre pro-} fesores y estudiantes de cooperación, en respeto de la autonomía estudiantil [...] el Consejo de estudiantes debe actuar con idéntica constancia que el Consejo de Profesores, tener derecho de insinuar ante este, proyectos que afecten la integridad del instituto haciéndose representar para ello por medio de voceros que lleven instrucciones precisas en cada caso. (Citado en Cuneo, 1988, p. 210)

Al igual que en el caso de la UNAM, la defensa de la autonomía universitaria cuestiona la supremacía del Estado y de los partidos e ideologías políticas, pues se trata de lograr un gobierno para sí misma, de establecer sus propias leyes y decisiones.

Aunque los desarrollos en las concepciones de autonomía eran interesantes, no lograron tener eco en la política pública pues a cada intento por instaurar la autonomía universitaria se respondía con la Constitución y

8 Arciniegas fue líder estudiantil, cofundador de la Federación de Estudiantes de Colombia, creada en 1921 con la clara convicción de defender la autonomía universitaria y la libertad de cátedra. 
el Concordato en mano para demostrar su invalidez. Ejemplo de ello fue la propuesta de reforma universitaria presentada en 1932 por Germán Arciniegas ante el Congreso de la República, en la cual ponía de relieve la financiación estatal adecuada, sin que esta interfiriera en la libre determinación administrativa, académica y del gobierno universitario. La segunda propuesta fue presentada en 1935, bajo el programa de la Revolución en Marcha de Alfonso López Pumarejo, el cual establecía la autonomía universitaria a través de la Ley 68 de 1935. Todas estas propuestas fueron desechadas una vez llegaban al poder gobiernos conservadores, por ello se modificó la ley en mención, durante el mandato de Laureano Gómez (1950- 1954). Este vaivén partidista no permitió un desarrollo de la concepción de educación pública ni de autonomía universitaria, pues como bien lo mencionaría Gerardo Molina, rector de la Universidad Nacional (1944-1948), en ella se instauró una autonomía relativa:

El gobierno financia la universidad, pero en su máximo organismo rector, el consejo directivo, participa con 3 de los 9 miembros. Cinco de ellos provendrán directamente de los gremios universitarios (decanos, profesores, alumnos); en cuanto a la elección del rector participan el presidente de la República y, de forma limitada, el consejo directivo. (Citado en Lucio \& Serrano, 1992, p. 39)

Debido a este déficit en la autonomía, el inicio de la década de los sesenta tuvo una importante presión estudiantil, gracias a la cual se dio lugar a la Ley 65 de 1963, en la que se concebía en el artículo $4 .^{\circ}$ la autonomía universitaria como:

[...] la capacidad jurídica y económica de la Universidad para organizarse, gobernarse y dictar las normas y reglamentos académicos, docentes y administrativos conforme a los cuales se realicen los fines que le son propios, dentro de la órbita constitucional y legal, y respetando las obligaciones contraídas por el Estado mediante tratos públicos. (Lucio \& Serrano, 1992, p. 59)

Estos alcances de la autonomía siguen siendo limitados por el lugar que ocupa el Ejecutivo en las decisiones de la universidad.

Con la CPC de 1991, no son muy notorias las modificaciones a esta concepción de autonomía, pues en su artículo 69 la define como la posibilidad de "darse sus directivas y regirse por sus propios estatutos, de acuerdo con la ley. La ley establecerá un régimen especial para las universidades del Estado [...]", pero en realidad los 
gobiernos y las decisiones universitarias están mayoritariamente en manos del Gobierno. A ello se suma que la jurisprudencia sobre la autonomía universitaria no establece diferencias entre la autonomía para universidades públicas y privadas (Villamil, s. f.).

Hasta la fecha el debate sobre la autonomía ha permanecido irresuelto, puesto que aún es evidente la intervención del Estado en las decisiones políticas como en el gobierno universitario tanto en la UNAM Como en la UN.
Sin embargo, son considerables las diferencias con respecto a la participación de la comunidad universitaria en los máximos órganos de gobierno, pues como lo ilustra Leopoldo Múnera (2011), en la Universidad Nacional el déficit de un gobierno propio se expresa en la mayor participación del Gobierno nacional y de otros agentes externos a la universidad, con respecto a la de la misma comunidad universitaria, a diferencia de lo que ocurre en la UNAM:

\begin{tabular}{|c|c|c|}
\hline Universidad & UNAM (Ley Orgánica de 1945) & $\begin{array}{c}\text { Universidad Nacional (Ley 30/1992, } \\
\text { Decreto 1210/1993 } \\
\text { y Acuerdo 011/2005) }\end{array}$ \\
\hline \multirow{2}{*}{$\begin{array}{l}\text { Cuerpos } \\
\text { colegiados } \\
\text { superiores }\end{array}$} & $\begin{array}{l}\text { Consejo Universitario: } \\
\text { Directivos universitarios: } 41 \% \\
\text { Representación profesoral: } 39 \% \\
\text { Representación estudiantil: } 19 \% \\
\text { Representación de los trabajadores y } \\
\text { administrativos: } 0,9 \% \\
\text { Comunidad universitaria: } 100 \% \\
\text { Representantes de la comunidad } \\
\text { universitaria: } 59 \%\end{array}$ & $\begin{array}{l}\text { Consejo Superior } \\
\text { Directivos Universitarios: } 12,5 \% \\
\text { Gobierno nacional: } 37,5 \% \\
\text { Representación profesoral: } 12,5 \% \\
\text { Representación estudiantil: } 12,5 \% \\
\text { Otros: } 25,0 \% \\
\text { Comunidad universitaria: } 37,5 \% \\
\text { Representantes de la comunidad } \\
\text { universitaria: } 25 \%\end{array}$ \\
\hline & $\begin{array}{l}\text { Junta de Gobierno: } \\
\text { 100\% nombrados directamente por el } \\
\text { Consejo Universitario }\end{array}$ & $\begin{array}{l}\text { Consejo Académico: } \\
\text { Directivos universitarios : } 87,8 \% \\
\text { Representación profesoral: } 6,1 \% \\
\text { Representación estudiantil: } 6,1 \% \\
\text { Comunidad universitaria: } 100 \% \\
\text { Representantes de la comunidad } \\
\text { universitaria: } 12,2 \%\end{array}$ \\
\hline
\end{tabular}

Fuente: Múnera (2011).

De allí se puede concluir que la principal diferencia entre la autonomía lograda en la UNAM y la Universidad Nacional radica en que en los órganos del gobierno universitario de la UNAM, como la Junta de Gobierno y el Consejo Universitario, no se designan representantes del Gobierno o de los sectores económico, productivo o empresarial. La Junta está confor- mada por quince miembros de la comunidad académica, los cuales son elegidos por el Consejo Universitario. Si bien ha existido una incidencia significativa por parte del Ejecutivo en las designaciones del rector - decisión que recae en la Junta de Gobierno-, y en algunas decisiones de la universidad, aún se concibe que el gobierno universitario 
debe estar orientado por la propia comunidad universitaria. De acuerdo con el análisis de Ordorika (2006) "Con variaciones históricas, el gobierno concedió a la unam un estatuto autónomo, los derechos legales de administrar sus recursos, de tomar decisiones académicas y de nombrar a sus autoridades" (p. 35). El caso colombiano es distinto pues, como vemos, desde antaño ha tenido entre los máximos órganos del gobierno universitario representantes del Ejecutivo. En la actualidad, el ministro de Educación preside el Consejo Superior, que está conformado, además, por representantes de los gobiernos local y regional, así como del sector productivo. Este elemento es muestra de la restringida capacidad de autogobierno e independencia en la toma de decisiones de las universidades públicas frente al Estado.

Este rasgo de la autonomía se refiere entonces a la lucha por un gobierno propio como garante de la independencia en las decisiones que atañen a la vida universitaria. Entendiendo que la razón de ser de la universidad está apoyada en el desarrollo del conocimiento y en los procesos formativos que contribuyen en la consolidación de proyectos sociales más justos, esta misión no responde al uso de la fuerza del Estado, de allí que se distancie rotundamente de la intromisión del mismo en su gobierno.

\section{La autonomía es a la universidad lo que la soberanía a los pueblos}

La última antinomia se dibuja perfectamente en el interés privado representado en el mercado y el actual modelo de desarrollo económico y su injerencia en la vida universitaria. Está intromisión no se puede entender por fuera de las reformas que han transitado el ámbito educativo durante las tres últimas décadas, las cuales se han orientado a la desfinanciación y descentralización, la evaluación de la calidad para condicionar el financiamiento educativo y la competitividad a escala mundial (Martinic, 2001). Desde esta perspectiva, la amenaza a la autonomía se expresa en las directrices de la banca multilateral (BM, OCDE, FMI), las cuales son pensadas en función de la rentabilidad de un "servicio", desdibujando la labor histórica de la universidad de contribuir en la construcción de los proyectos y de la soberanía de los pueblos. 
En los casos estudiados, desde la década de los ochenta ${ }^{9}$ con las medidas de ajuste estructural se buscó modificar el modelo de financiación de la UNAM y de la Universidad Nacional de Colombia. Estas medidas incidieron en la disminución del presupuesto universitario; para el caso de la UnAm se pasó de 17000 mi-llones que tenía en 1982 a 10826 millones en 1989 (Díaz Barriga, 1999), y en Colombia, en este mismo año, a través del Decreto 728, se estableció que el Gobierno solamente financiara el $90 \%$ del funcionamiento de las universidades oficiales, política que para 1990 se redujo al 70\% (Lucio \& Serrano, 1992) y sigue disminuyendo.

9 Sin embargo, en Colombia encontramos desde la década de los sesenta varias amenazas en la definición de los destinos de las universidades públicas. Al respecto se puede mencionar el Plan Atcón (1961) y el Plan Básico (1966). En ambos casos se violentaba la autonomía universitaria pues estos planes representaban la intervención estadounidense en la universidad pública. Este señalamiento se sustenta en que el primer informe cuestionaba la estructura, los contenidos y las formas de enseñar en la universidad; incluso se llegó a recomendar que se estableciera "una universidad legalmente independiente y privada, organizada gerencialmente por departamentos (según el modelo norteamericano), y en el cual hay una división funcional muy precisa entre los diversos estamentos; a unos les corresponde administrar, a otros enseñar, y a los estudiantes estudiar (no gobernar ni administrar)". De igual manera el Plan Básico de la Educación Superior coordinado por la Misión California (1966-1967), en colaboración con la Asociación Colombiana de Universidades, Ascun, y la fun, daba continuidad al plan Atcón. Este se cuestionaba por qué las universidades y sus directivos fueron incapaces de manejar sus propios asuntos delegando para ello una comisión designada por el Gobierno nacional y Consejo Nacional de Educación Superior, violentando así la autonomía universitaria. (Véase Lucio \& Serrano, 1992).
A ello se suma que las políticas aperturistas de los noventa, junto con la firma del Tratado de Libre Comercio con Norteamérica en 1994, y el TLC en Colombia en 2014, han contribuido en los procesos de privatización de la educación y de la educación superior por doble vía: de un lado los Estados mancomunadamente con la banca multilateral han entregado al capital privado gran parte de la responsabilidad de financiación de la educación pública, creando políticas de autofinanciación o cofinanciación con el capital privado. Por otra parte, se reduce la ampliación de las universidades públicas, lo que permite a su vez la proliferación de una demanda mayoritariamente privada. De allí que en las últimas décadas no se encuentra la aparición significativa de nuevas universidades públicas, en su defecto se obliga a ampliar la cobertura con los mismos recursos e infraestructuras, por ejemplo.

Es claro que la autonomía se ve violentada gravemente al entregar a fondos privados las responsabilidades de la educación pública, tal como lo expresa Aboites, a propósito de la incidencia del TLCN en el la educación en México:

En nuestro país la autonomía se elude también mediante la existencia de los fondos de financiamiento, como el mencionado fOMEs -Fondo de Modernización para la Educación Superior-. Con esto se ha colocado a 
las universidades en un grado mayor de dependencia respecto de los criterios impulsados por el gobierno para la educación superior. (1999, p. 243)

De esta manera, al entregar a un fondo privado el funcionamiento de las universidades se generan altos grados de dependencia que obligan a las propias universidades a rendir cuentas y a responder a los intereses privados.

Así vemos que este proceso atenta contra la autonomía universitaria, específicamente en que es desde el mercado que se define qué conocimientos se producen, qué investigar y qué conocimiento circula en las universidades públicas. Acosta (2008) menciona que este proceso transforma de manera silenciosa el significado de la autonomía universitaria, en la medida en que "se dejan de lado principios como el autogobierno y la libertad de distribución de los recursos públicos para instaurar una autonomía basada en la producción de resultados y el establecimiento de la diferenciación y la diversificación de los recursos financieros" (Acosta, 2008, p. 36).

Esto se observa especialmente en la lógica de los TLC que para poder poner en funcionamiento el mercado educativo en el ámbito internacional pregonan la unificación de currículos, núcleos comunes y programas de pregrado, así,

Con el comercio transfronterizo, una de las implicaciones más serias es que la historia y necesidades de la práctica profesional de un país ya no son los elementos fundamentales de la definición del contenido de una profesión (a nivel incluso de currículo) sino el proceso de homologación con los otros dos países, especialmente con los EeUu. (Aboites, 1999, p. 30)

Para el caso colombiano

[...] el ingreso masivo de universidades empresariales y por franquicia serían el certificado de defunción para nuestras comunidades académicas; la nueva universidad produciría eficientemente mercancías académicas efímeras o simplemente útiles, pero frenaría el desarrollo de las capacidades de innovación que no estuvieran ligadas inmediatamente a la rentabilidad económica. Las conquistas culturales conseguidas con la universidad tradicional saltarían en el aire ante el paso arrollador del capitalismo académico. (Múnera, 2006, p. 112) 
Ello implica que los contenidos curriculares y las necesidades de formación ya no respondan a los contextos nacionales sino a los intereses del mercado educativo transfronterizo. Este problema se amplía con las políticas de evaluación de la calidad, pues muchas de ellas responden a las exigencias mercantiles, tal como lo expone el profesor Múnera:

Desde luego, las restricciones a la autonomía no se limitan al gobierno de las instituciones, sino que se extienden a la producción misma del conocimiento. La evaluación mediante indicadores determinados en virtud del mercado de la educación, pasa al orden del día. El Banco Mundial nos advierte que gracias a estos mecanismos de rendimientos de cuentas los administradores de las instituciones serán obligados a tomar las decisiones que hasta ahora han estado evadiendo, por ejemplo, reasignar los recursos en respuesta a las necesidades de los clientes y los consumidores. $(2007$, p. 108)

Es evidente que las lógicas de la evaluación y la acreditación de programas e instituciones de educación superior no gozan de independencia y terminan respondiendo a una eva- luación acrítica, con indicadores que desvirtúan la naturaleza misma de la universidad pública, que además hacen de los encargos misionales (docencia, extensión e investigación) un fortín que debe luchar por "recursos privados o los recursos públicos asignados en función de criterios privados" (Múnera, 2007, p. 108). De esta manera se pierde el lugar de las universidades en el desarrollo científico y de pensamiento crítico de nuestros pueblos, y deja de ser un interlocutor válido para las sociedades contemporáneas.

La autonomía universitaria se opone entonces al capitalismo académico, representado en la producción de investigación, conocimiento y programas de extensión que resulten rentables económicamente y que atiendan las necesidades del capital (Múnera, 2006, p. 137). En cambio nuestra autonomía, desde el lugar de lo público, se sigue preguntando por la razón de ser de la universidad en nuestros pueblos, en la consolidación de proyectos sociales alternativos, capaces de resistir la naturalización del capitalismo. Parafraseando a Aboites, la autonomía sigue representando zonas de poder alternativas a un poder central, el capitalista, en relación con el conocimiento. Para eso sirve pensar la autonomía universitaria hoy, porque ella representa la soberanía de la universidad al abordar el conocimiento desde el cual se definen proyectos profesionales para orientar y construir el bien común. 


\section{Un rediseño de la autonomía universitaria}

El argumento que se sostiene es que la autonomía universitaria desde las universidades públicas surge de la disputa con aquello que representa un interés privado identificado en un credo, una ideología, forma de gobierno o en el mercado. Por ello es necesario reconocer esta configuración de la autonomía universitaria, sin el ánimo de mitificarla románticamente, tal vez con la idea de contribuir a su rediseño.

Para ello es necesario resaltar que la relación entre universidad y Estado es una relación necesaria pero que no puede supeditar el trabajo académico, investigativo y de producción de conocimiento a los intereses del último, como diría Boaventura de Sousa Santos (2007) a propósito de la autonomía universitaria "De manera semejante a lo que pasa con el sistema judicial, donde la independencia de los tribunales no es puesta en discusión por el hecho de ser financiados por el Estado" (p. 24), las universidades públicas no pueden ser cuestionadas por ser financiadas estatalmente, máxime cuando los recursos del Estado provienen en buena medida de nosotros, los contribuyentes. Son nuestros recursos los que garantizarían la existencia e independencia de la universidad pública y el Estado estaría Ilamado a retribuir de alguna manera estos ingresos.

Ello implicaría andar una autonomía que piense en los proyectos de sociedad, en la soberanía de los pueblos, en los problemas de la desigualdad y la exclusión social, que se exprese en la libertad de asociación con otras instituciones a nivel mundial, no por sus relaciones mercantiles sino por las potencias que pueden encontrarse para responder a las demandas de los pueblos (indígenas, afrodescendientes, urbanos, campesinos, mujeres, jóvenes), puesto que no podemos seguir anclados en una universidad solo urbana y que excluye a la mayor población juvenil.

De igual manera esta autonomía universitaria al estar expresada en "la capacidad de la universidad para decidir sobre sus destinos", no puede dejar por fuera el pensar la formación de sus profesionales en relación con el mundo que nos queda por vivir, ello implica una universidad comprometida con otras alternativas epistémicas y económicas. 
Hablar de autonomía universitaria no es un indicativo de aislamiento y ruptura con el Estado, máxime cuando esta ha sido una relación histórica, por ello

[...] es importante reiterar que la autonomía es una relación que se establece entre la universidad, el resto de las instituciones del Estado y la sociedad. Esta relación es histórica en un doble sentido. En primer lugar porque ha sido una condición constitutiva de la universidad a lo largo de buena parte de su existencia. En segundo término, porque se transforma $y$ se recrea a lo largo de la historia, a partir de la interacción de actores, sujetos sociales e instituciones; del devenir mismo de la universidad en el desempeño de sus funciones y el desarrollo de sus dinámicas y conflictos internos; así como en la articulación con la Nación, el Estado y la sociedad. (Ordorika, 2010)

Ello implica que no podemos resignificar la autonomía hoy por fuera de los proyectos de sociedad que queremos construir junto a la universidad.

\section{Referencias bibliográficas}

Aboites, H (1999). Viento del Norte. TLC y privatización de la educación superior en México. México: Universidad Autónoma Metropolitana, Unidad Xochimilco/Plaza y Valdés.

Acosta S., A. (2008). La universidad en América Latina: problemas, desafíos y temas capitales. Universidades, 58 (36), 69-82.

Aguilera M., A. (2010). A 200 años de herencias y herejías en la universidad pública colombiana. Revista Colombiana de Educación, 59, 206-226.

Aguilera M., A. (2011). Entre bastidores: la memoria larga de la universidad pública (ColombiaMéxico). Pedagogía y Saberes, 34, 67-83.

Aguilera M., A. (2013). Subjetividades políticas en movimiento(s). La defensa de la universidad pública Colombia-México. Bogotá. UPNEditorial Magisterio.

Gaceta de Colombia. (1826, diciembre 24). Anuncio de instalación de la Universidad Central y de la Academia Nacional (1826). En: M. Aguilera P., (2001). Universidad Nacional de Colombia: génesis y reconstitución. Serie Historia de la Universidad Nacional de Colombia. UN, Unilibros. 
Gaceta de Colombia. (1828, octubre 3). Simón Bolívar modifica plan de estudios por participación de estudiantes en la conspiración septembrina. En: M. Aguilera P., (2001). Universidad Nacional de Colombia: génesis y reconstitución. Serie Historia de la Universidad Nacional de Colombia. UN, Unilibros.

Alvarado, M. (1999). La universidad libre: primer movimiento estudiantil del México independiente (1875). En: R. Marsiske (coordinadora). Movimientos estudiantiles en la historia de América Latina. unAm-CESU, Plaza y Valdés Editores. México. Alvarado, M. (2001). La Universidad del siglo xix. En: R. Marsiske (coord.). La universidad de México. Un recorrido histórico de la época colonial al presente. México: unAm-CESU/Plaza y Valdés.

Archila, M. (1999). Entre la academia y la política: el movimiento estudiantil en Colombia 1920-1974. En: R. Marsiske (coord.). Movimientos estudiantiles en la historia de América Latina (pp. 158-175). México: unAm-CESU/Plaza y Valdés.

Arendt, H. (1993). La condición humana. Barcelona: Paidós.

Camboni, S. (1998). Universidad, sociedad y cambio tecnológico. En: R. Rodríguez y H. Casanova (comps.). Universidad contemporánea. Racionalidad política y vinculación social (tomo I, pp. 87-109). México: Cesu-Porrúa.

Condorcet, J, A. (2007). Cinco memorias sobre la instrucción pública. Buenos Aires: El Signo.

Cúneo, D. (comp.) ([1978]/1988). La reforma universitaria. Caracas: Biblioteca Ayacucho.

Díaz Barriga, A. (1999). Contexto nacional y políticas públicas para la educación superior en México 1950-1995. En: H. Casanova y R. Rodríguez (coords.), Universidad contemporánea. Política y gobierno (tomo II, pp. 371-386). México: CESU/Porrúa.

García Cantú, G. (1988). Historia en voz alta: la universidad. México: UNAM.

González G., E. (2001). La Real Universidad. En: R. Marsiske (coord.). La universidad de México. Un recorrido histórico de la época colonial al presente. CESU-Plaza y Valdés Editores. UNAM.

Hernández de Alba, G. (2009). Documentos para la historia de la educación en Colombia. Traducción tomada de la obra, tomo 4 (1767-1776), pp. 195-227. Bogotá, 1980. Revista Aquelarre, 8 (16). 
Levy, D. (1995). La educación superior y el Estado en Latinoamérica. Desafíos privados al predominio público. México: CeSU-UNAM.

Lucio, R. \& Serrano, M. (1992). La educación superior, tendencias y políticas estatales. Bogotá: lepri-un/Tercer Mundo.

Manifiesto de Córdoba, Argentina. ([1918] 1978). En: Dard, C.: La Reforma Universitaria (19181930) (pp. 3-7). Caracas: Biblioteca Ayacucho.

Marsiske, R. (coord.). (2001). La universidad de México. Un recorrido histórico de la época colonial al presente. CEsu-Plaza y Valdés Editores-unam.

Martinic, S. (2001). Conflictos políticos e interacciones comunicativas en las reformas educativas en América Latina. Revista OEI, 27.

Múnera, L. (2006). El gobierno universitario. En: Documento de Trabajo N. ${ }^{\circ}$ 1. Debates sobre la Educación Superior. Bogotá: Planeta Paz.

Múnera, L. (2007). ¿Hacia dónde va la universidad pública? Tendencias globales en política pública para la educación superior. En: Documento de Trabajo N. ${ }^{\circ} 1$. Debates sobre la Educación Superior. Bogotá: Planeta Paz.

Múnera, L. (2011). Ecos de una audiencia pública. Mimeo. Bogotá.

Ordorika, I. (2006). La disputa por el campus. Poder, política y autonomía en la UNAM. México D. F.: CESU, unAm, Plaza y Valdés.
Ordorika, I. (2010) La autonomía universitaria. Una perspectiva política. En: Perfiles Educativos. 21. Número especial. Rabotnikof, N. (1997). El espacio público y la democracia moderna. México.

Ramírez, G. (2001). La Universidad Autónoma de México (19331944). En: R. Marsiske, (coord.). La universidad de México. Un recorrido histórico de la época colonial al presente. México: UNAM-CESU / Plaza y Valdés.

Santos, B. de Sousa. (2007). La universidad del siglo xxı. La Paz: Cides-UMSA.

Sierra, J. (1891). Obras completas, tomo 5. Discursos. El discurso sobre "Organización de la enseñanza pública".

Sierra, J. (1910, 26 de abril). Obras completas, tomo 5. Discursos. "Iniciativas para crear la Universidad".

Soto Arango, D. (1999). El movimiento de estudiantes y catedráticos en Santa fe de Bogotá a finales del siglo xvIII. En: R. Marsiske (coord.). Movimientos estudiantiles en la historia de América Latina. México: UNAMCESU, Plaza y Valdés.

Tunnerman, C. (1976). La investigación en la universidad latinoamericana. En Deslinde, Cuadernos de Cultura Política Universitaria, 75. UNAM 
Varela Barrios, E. (2005). Dimensiones actuales de lo público. A propósito de las interrelaciones entre Estado, Management y Sociedad. Pensamiento y Gestión, 18, 37-68.

Villamil Ardila, C. (s. f.). Alcance de la autonomía universitaria en Colombia, 1980-2002. Una reflexión desde la evolución legislativa y jurisprudencial. 